\title{
Un réseau social pour le FLE. Quelles perceptions et quels usages de l'apprenant?
}

Jean-François Grassin

\section{(2) OpenEdition}

Journals

Édition électronique

URL : http://journals.openedition.org/rdlc/1588

DOI : $10.4000 /$ rdlc. 1588

ISSN : 1958-5772

Éditeur

ACEDLE

Référence électronique

Jean-François Grassin, « Un réseau social pour le FLE. Quelles perceptions et quels usages de l'apprenant? », Recherches en didactique des langues et des cultures [En ligne], 10-2 | 2013, mis en ligne le 07 juin 2013, consulté le 30 avril 2019. URL : http://journals.openedition.org/rdlc/1588 ; DOI $10.4000 /$ rdlc. 1588

Ce document a été généré automatiquement le 30 avril 2019

\section{cc) (†)}

Recherches en didactique des langues et des cultures is licensed under a Creative Commons AttributionNonCommercial-NoDerivatives 4.0 International License 


\title{
Un réseau social pour le FLE. Quelles perceptions et quels usages de l'apprenant?
}

\author{
Jean-François Grassin
}

\section{Introduction}

1 Cette étude porte sur l'intégration d'un Réseau Social Numérique (RSN) public au sein d'une institution d'enseignement du Français Langue Etrangère (FLE) à l'université en France. Les RSN combinent des pages de profils individuels à des outils collectifs d'interaction, tels que chat, blog et forum de discussion. D'un point de vue pédagogique, de tels outils permettent d'accroître le potentiel des interactions en dehors du temps de classe traditionnel, de fournir un input langagier authentique et contextualisé et un espace pour des interactions potentiellement signifiantes, dans une interface à l'architecture qui repose sur la mise en contact des membres et qui incite à la participation.

2 Cette étude souhaite confronter les potentialités des RSN pour l'apprentissage d'une langue à une réalisation située, dans une démarche écologique. Nous cherchons à identifier les conditions de l'actualisation de ces potentialités. Dans cet article, nous rendons compte d'une expérimentation d'une durée d'un semestre. Nous examinerons les usages que font les étudiants de ce réseau social et leurs représentations de ce site reliées à leur apprentissage de la langue.

3 Pour commencer, nous donnerons quelques définitions du web 2.0 et des réseaux sociaux en usage, ainsi qu'une approche de leur appropriation en contexte d'apprentissage. Après avoir décrit le contexte de l'étude et sa méthodologie, nous analyserons usages et représentations, et tracerons des perspectives pour la suite de notre recherche et l'évolution du dispositif proposé. 


\section{Un cadre théorique à l'articulation de champs disciplinaires divers}

\section{Web 2.0 et web social}

4 Le terme web 2.0 fut proposé pour la première fois par O’Reilly et son équipe en 2004. Il s'agit d'un macro-concept désignant un ensemble de pratiques et de principes. Le web 1.0 suivait une logique d'auteurs/lecteurs, une logique de publication unidirectionnelle (ou " one-to-many"), alors que le web 2.0 suit une logique "lecture/écriture" interactive. Il met l'accent sur la participation, la connectivité, la collaboration, le partage de connaissance et d'idées entre utilisateurs (Lee \& Mc Loughlin, 2010).

5 Le web 2.0 se réfère à "la plateforme technologique permettant aux applications des réseaux sociaux de se mettre en œuvre grâce aux possibilités qu'ont les utilisateurs de créer, distribuer, partager et manipuler différents types de contenus, la plupart accessibles à tous librement" (Zourou, $2012: 12$ ), et en ce sens, permet un web "social ".

\section{RSN et Web social}

6 Sous-ensemble des médias sociaux en tant que services qui prennent en charge les interactions de groupe ("software that support group interaction", Shirky (2003) repris par Lee \& Mc Loughlin, (2010) les RSN, selon Stenger et Coutant (2010 : 221), constituent des services web qui permettent aux individus :

1. de construire un profil au sein d'un système ;

2. de gérer des listes d'utilisateurs avec lesquels ils partagent un lien;

3. de naviguer sur leur liste de liens et sur ceux établis par les autres.

7 Ces RSN fondent leur attractivité essentiellement sur les trois premiers points et non sur une activité particulière.

8 S'il est donc essentiel de retenir la différence entre les sites dont le réseau est principal et ceux pour lesquels il est secondaire, tous les sites intègrent peu à peu les mêmes fonctionnalités. Stenger et Coutant (2010) proposent d'adopter une dichotomie plus précise en fonction des usages et des buts de l'activité en ligne où l'on aurait d'un côté les médias dont l'activité principale est "la computation sociale", activité de partage et de commentaire de fichiers, ou "object-centered sociality", et d'un autre, les RSN qui " focalisent l'activité sur la création et la mise en scène de cette face publique (Goffman, 1974) que constitue le profil" (ibid., 221). Ito et al. (2008) ont distingué ainsi deux orientations participatives qu'elles nomment "interest-driven activity", où les activités sont orientées autour d'un intérêt, d'une passion, et la "friendship-driven activity", orientée vers l'amitié en ligne. Boyd et Ellison (2007) avaient déjà pointé cette différence lorsqu'elles affirmaient que "les RSN sont d'abord organisés autour des personnes, pas des intérêts". La structuration de l'activité ne se fait pas autour de sujets mais autour d'individus, au centre de la communauté.

9 Une question importante pour nous est de savoir quelles représentations du site proposé se forment chez les étudiants, notamment pour nous permettre de déterminer son acceptabilité et son utilité (Tricot, 2011 : 181) pour un enseignement/apprentissage de la langue. Ces représentations se construisent de manière située, socialement et dans 
l'action, mais aussi historiquement, au travers de la culture numérique préalable des acteurs de la situation.

\section{RSN et communauté d'apprentissage}

10 Ce qui diffère entre "réseau social" et "communauté" est la dynamique des liens sociaux Ciussi (2007). Pour Henri et Pudelko (2006), la communauté se caractérise par un ensemble de liens forts, "la force du lien social qui unit ses membres qui ont un centre d'intérêt partagé". La force des liens est une densité signifiante. Plus le lien est fort, plus les échanges portent une charge émotionnelle intense (Granovetter, 1973). Le terme de "communauté" préjugerait donc -de manière probablement un peu optimiste, selon Mangenot (2011)- de l'établissement de liens sociaux forts et d'une certaine interdépendance entre les membres.

11 Cependant, c'est oublier que, en ligne, nombre de communautés reposent sur la " coopération des liens faibles" (Cardon 2008). Cardon remarque qu'il est souvent abusif de parler de "communauté" sur le web car il s'agit plus souvent d'engagements collectifs " provisoires, imparfaits et labiles", qui se caractérisent aussi par "une très grande hétérogénéité des formes de participation", ce qui lui permet de dire que "les communautés ne sont que des réseaux solidifiés". Nous pourrions donc soutenir qu'un RSN n'est pas une communauté virtuelle mais "un terreau sur lequel peuvent émerger des communautés" (Stenger \& Coutant, $2010: 222$ ).

Le sentiment d'appartenance à une communauté repose sur deux concepts clés : l'interdépendance et les relations interpersonnelles à l'intérieur de cette communauté. D'une part, l'interdépendance entre les individus implique que le groupe confère une valeur, le respect, l'attention à l'individu et, parallèlement, par le temps et l'investissement concédé, l'individu donne valeur au groupe. D'autre part, les relations interpersonnelles, relations entre pairs comme celles avec les enseignants renforcent le sentiment de communauté. En effet, l'émergence d'une communauté repose sur celle d'une identité collective correspondante, elle-même se forgeant par le biais des liens sociaux émanant des interactions. La notion d'identité est construite par les échanges socio-affectifs et socio-cognitifs qui créent des liens sociaux de plus en plus denses. C'est toujours affaire de processus, le type de liens évolue dans le temps et selon les activités et "le maillage social (formes et processus) tend ainsi à constamment varier car il est co-construit par l'activité de ses membres" (Cuissi, 2007 : 4).

13 Cependant, dans une formation hybride en langue, on peut penser que les liens forts ne vont pas forcément se construire en premier lieu au sein du RSN mais en face-à-face. Ainsi, être membre d'une communauté n'entraîne pas forcément un haut degré d'engagement et une participation active sur la plateforme numérique. Nous suivons en cela la remarque de Zourou (2012) :

Loin d'un enthousiasme technophile qui, par définition, associe communautés et réseaux sociaux à un haut degré d'engagement, de participation et d'activité conjointe, nous prônons une attitude plus réaliste qui cherche à comprendre la nature et les types de pratiques interactionnelle qui peuvent émerger dans ces espaces (Zourou, $2012: 12$ ).

14 Si l'utilisation du site que propose le dispositif d'enseignement/apprentissage que nous étudions, repose a priori sur des buts et des intentions d'apprentissage préexistants, nous cherchons à voir si d'autres usages, hétérogènes, imprévus et changeants, ne vont pas émerger. 


\section{Les traces de la présence sociale comme indicateurs de la dynamique sociale en construction}

15 Un pré-requis à une situation d'apprentissage efficace semble être la présence sociale (Garrison, Anderson \& Archer, 2000) qui peut se définir comme "la capacité des participants à projeter leurs caractéristiques personnelles dans la communauté ainsi que de se présenter aux autres participants comme des personnes réelles" (Garrison et al., $2000: 89$ ). Elle se repère à travers des indicateurs de réactions affectives, d'ouverture à la communication et de réactions de cohésion par rapport au groupe, dans les interactions avec l'enseignant comme avec les pairs. Pour Anis (1998) les liens forts correspondent à un usage "horizontal de discussion", alors que les liens faibles correspondent à ce qu'il appelle une "verticalité informationnelle".

Pour analyser les traces de la présence sociale au travers des productions et des interactions écrites, nous reprendrons ici le schéma de Ciussi (2007) qui propose une analyse des interactions par phases d'évolution socio-discursives.

Figure - phases d'évolution socio-discursives (Cuissi, 2007)

\section{Echanges socio-affectifs $\longrightarrow$ Echanges socio-cognitifs}

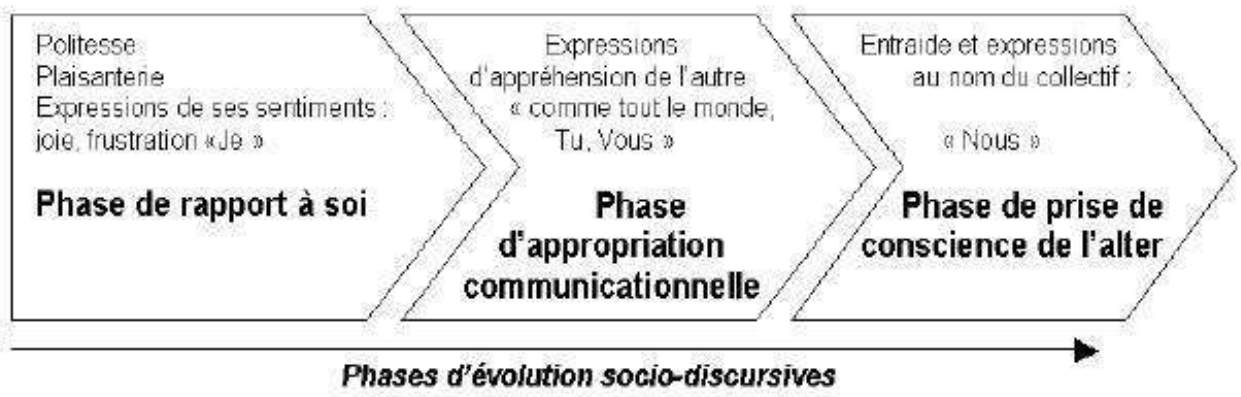

17 Les trois phases que repèrent Ciussi peuvent être rapprochées des trois phases que déterminent Pakdel et Springer (2010) et représentent trois aspects de la dynamique sociale :

- la première phase, phase de rapport à soi chez Ciussi, phase de dévoilement de soi chez les seconds auteurs se repère par l'emploi de mots faisant référence à soi-même, employés pour partager une opinion, un sentiment, un souvenir...

- la deuxième phase, celle d'appropriation communicationnelle est aussi celle de l'établissement de liens socio-affectifs (expressions d'appréhension de l'autre, termes d'adresse tu/vous)

- enfin, la dernière et troisième phase est celle de la prise de conscience de l'alter, qui correspond au développement d'une identité commune (expressions au nom du collectif, termes faisant référence explicitement ou implicitement au groupe ou à ces membres).

Dans ces traces de participation, nous cherchons la dynamique sociale en construction. 


\section{Pour une articulation du formel et de l'informel}

19 En contexte homoglotte, la plupart des expériences d'apprentissage relève d'un mélange d'apprentissage formel et d'apprentissage informel. Le tableau ci-dessous reprend les caractéristiques des trois types de situations ${ }^{1}$ :

Tableau 1 - Formel, non-formel, informel

\begin{tabular}{|l|l|}
\hline Types & caractéristiques \\
\hline $\begin{array}{l}\text { Apprentissage } \\
\text { formel }\end{array}$ & $\begin{array}{l}\text { Institutionnel, objectifs, durée et soutien fournis, intentionnel et } \\
\text { certification }\end{array}$ \\
\hline $\begin{array}{l}\text { Apprentissage non } \\
\text { formel }\end{array}$ & $\begin{array}{l}\text { Non institutionnel et sans certification; intentionnel avec objectifs, } \\
\text { délais et soutien structurés }\end{array}$ \\
\hline $\begin{array}{l}\text { Apprentissage } \\
\text { informel }\end{array}$ & $\begin{array}{l}\text { Résulte d'activités quotidiennes liées au travail, à la vie de famille et aux } \\
\text { loisirs; non structuré et non intentionnel }\end{array}$ \\
\hline
\end{tabular}

20 On se rend compte que le dispositif techno-pédagogique que représente le RSN amène à concevoir une porosité entre ces concepts. Le formel et l'informel ont tendance à être de plus en plus poreux grâce au web, de même que les sphères académiques et privées. Mangenot (2011) propose ainsi l'hyperonyme "appropriation" dont les situations peuvent se décrire sur un continuum qui irait de l' "acquisition" (situation informelle, par immersion) à l' "apprentissage" (situation formelle).

21 Il incombe au didacticien de réfléchir aux situations d'apprentissage qui peuvent émerger, intentionnellement ou non, d'un dispositif en ligne et aux cadres communicatifs et interactionnels qu'il propose, formels ou informels. La reprise d'un schéma proposé par Marcia Conner (1997-2013), ci-dessous, nous aide à voir ce qui est en jeu ici avec les outils du web 2.0. 


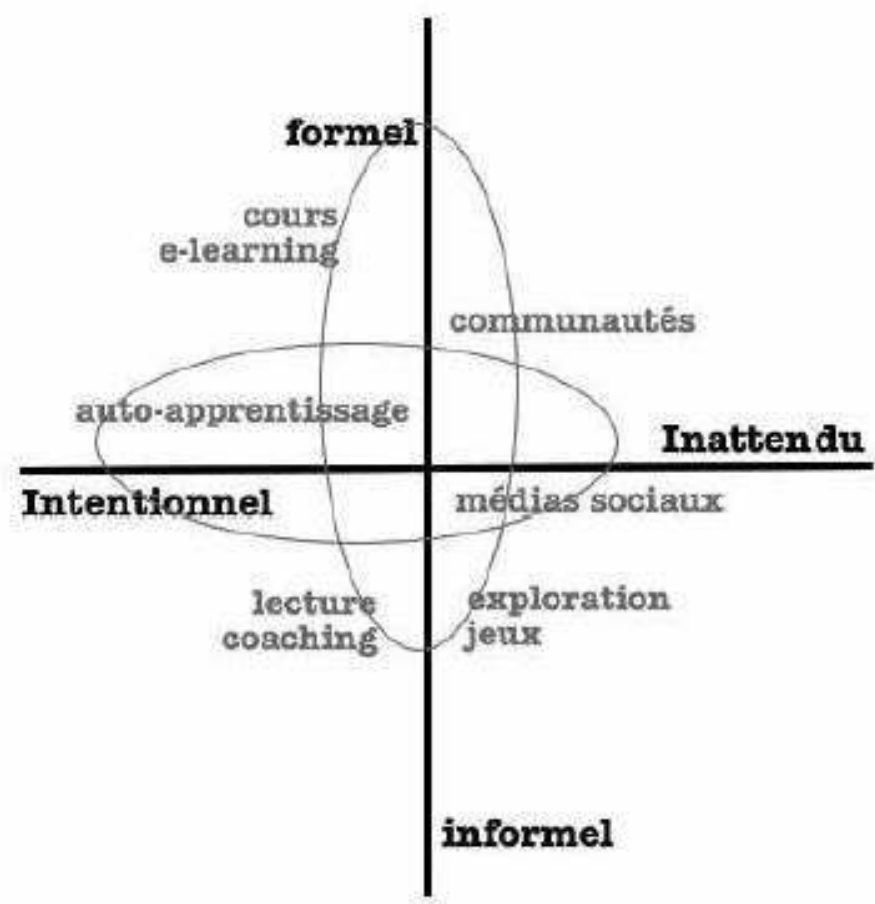

D’après Marcia L. Conner 2004-2009 d'apprentissage de la langue, comme un espace :

- formel d'apprentissage de la langue, mais non clos,

- laissant place à d'autres espaces potentiels de situations d'apprentissage non-formel, où l'apprenant fixe en partie les règles (objectifs et formes)

- ouvert vers l'apprentissage inattendu, restant ouvert sur le web.

Les espaces d'apprentissage sur le web permettent une porosité de ces espaces. Le web propose un réseau où ces espaces se côtoient et s'interpénètrent.

\section{L'appropriation du dispositif comme trajectoire située et distribuée}

Il faut considérer le réseau comme une technologie en action dans l'organisation et comme une technologie socialement construite. Dans ces conditions, pour qui veut analyser des usages situés, le concept d'appropriation est fructueux et incontournable. Guichon (2011) rappelle à juste titre qu'étudier les pratiques implique en particulier de prendre en compte les rapports que les individus développent vis-à-vis des objets et des logiques sociales qui façonnent les usages.

De nombreux auteurs (Arnold \& Paulus, 2010 ; Dabbagh et al., 2012) soulignent que les étudiants n'utilisent guère les avantages des technologies web 2.0 pour l'apprentissage formel. Ainsi, dans une étude de 2012, Wodzicki et al. notent qu'aux États-Unis, 96\% des utilisateurs de RSN le font pour rester en contact avec leurs amis alors que seulement $10 \%$ d'entre eux les utilisent pour des objectifs académiques. Le fait d'utiliser un RSN dans un cadre institutionnel implique une tension entre les deux pôles que constituent 
l'instrument mobilisé et les finalités ou les motifs de l'activité (Fluckiger, 2011). Dans un contexte scolaire, Guichon (2011) repère deux logiques à l'œuvre, qui peuvent être concurrentes ou complémentaires : une logique d'imposition et une logique d'appropriation.

La pluralité interne des sujets engagés dans l'activité de communication instrumentée (Fluckiger, 2011) - le sujet comme jeune, étudiant, apprenant...- va impliquer une pluralité des régimes d'engagement, au travers de négociation identitaires. Cette hypothèse de la discontinuité des pratiques invite à rechercher comment les mêmes outils peuvent faire l'objet de processus d'appropriation et de genèses instrumentales différents suivant les contextes d'usage (Fluckiger, 2011 ; Ito, 2008). Mais elle permet aussi d'envisager la coexistence de plusieurs logiques d'actions différentes, rejoignant en cela les approches situées de l'apprentissage et les approches socio-culturelles.

\section{Méthodologie}

\section{Fondements de la recherche}

L'apprentissage d'une langue étrangère ne se limite pas à l'intériorisation d'un système linguistique, culturel et social mais au développement de la capacité même de participer à une pratique sociale (Pekarek Doehler, 2000). Dans cette perspective, le développement de compétences langagières dépend non d'une centration sur la langue, mais de son utilisation instrumentale à des fins sociales et de la prise de responsabilité de l'apprenant dans la gestion du discours. Il s'agit d'envisager "la cognition humaine comme étant contextuellement déployée à l'intérieur d'activités sociales, qui débouche sur l'idée d'une cognition "située" et "distribuée" dans des contextes sociaux, institutionnels et interactifs." (Mondada \& Pekarek Doehler, 2000 : 5).

Notre posture de recherche envisage la nature émergente et contingente des contextes d'apprentissage et de la condition d'apprenant. L'approche socio-culturelle du développement cognitif amène, comme le soulignent Mondada \& Pekarek Doehler (2000 : 8), à une redéfinition radicale des compétences et des apprentissages en termes de participation à une communauté de pratique :

C'est dans les modes d'organisation de structures de participation que l'apprenant s'approprie non seulement des formes linguistiques mais aussi des savoir-faire communicationnels. Ainsi, les modes de participation [...] permettent de caractériser les occasions, les expériences, les contextes plus ou moins favorables à l'acquisition, plus ou moins passagers ou sédiments dans des lieux sociaux d'apprentissage (Mondada \& Pekarek Doehler, 2000 : 13).

La présente étude a été conçue pour identifier les usages du RSN en lien avec les activités proposées par les enseignants et en dehors des activités prescrites, et pour examiner la perception des étudiants des bénéfices associés à leur usage d'un RSN pour leur apprentissage du français. Il s'agit ici de rendre compte d'une étape exploratoire de la recherche.

Ainsi, pour répondre à notre question de recherche -à savoir dans quelle mesure ce réseau constitue-t-il un potentiel pour l'apprentissage des langues-, nous avons cherché, dans une première étape, à déterminer quels usages les étudiants font de ce réseau social numérique, d'une part et quelle est leur perception de ce réseau pour leur apprentissage de la langue française, d'autre part. En effet, l'étude des représentations sociales 
permet la prise en compte, du point de vue des acteurs, des systèmes de valeurs, des rapports de force, des biens symboliques qui circulent dans les communautés de pratiques, qui structurent les apprentissages et qui sont structurés par les pratiques et les apprentissages (Pekarek Doehler, 2000 : 8).

31 Ce sont les résultats de cette première étape que nous présentons ici.

\section{Présentation du terrain de recherche}

\section{Le contexte}

L'étude a été conduite dans le contexte d'un centre d'enseignement du français langue étrangère à l'université, le Centre International d'Etudes Françaises (CIEF) de l'Université Lumière Lyon 2. L'observation a porté sur 59 étudiants inscrits à un cours préparant à un diplôme universitaire de langue, le Diplôme universitaire d'Études Françaises (DUEF), à un niveau B1. Il s'agit d'une session de 10 semaines de cours dont 17 heures 30 de cours en face à face par semaine et un cours en ligne de 20 heures.

Cette soixantaine d'étudiants est répartie en 3 groupes. La majorité des étudiants est féminine (35 sur 59). La moyenne d'âge est de 24 ans (le plus âgé a 33 ans et le plus jeune, 19). 33 étudiants sont d'origine asiatique, $9 \mathrm{~d}$ 'Amérique du nord, $7 \mathrm{~d}$ 'Amérique latine et centrale, 7 d'Europe de l'est, 2 d'Afrique et une d'Europe du nord.

Le dispositif (cf. Figure 3) propose ainsi 2 modalités de travail aux étudiants : du présentiel et du à distance, mais en proportion très inégale. L'environnement numérique d'enseignement/apprentissage est constitué d'un RSN que nous décrivons ci-dessous. Le module en ligne, intitulé "Parcours en ligne", repose sur une approche par tâches. Les micro-tâches et les tâches intermédiaires sont auto-correctives. Seule, la tâche finale est à envoyer pour évaluation au tuteur avant d'être publiée sur le réseau social. Les tuteurs sont aussi enseignants des cours en présentiel, ceci afin de ne pas trop isoler du dispositif la partie en ligne.

Figure 3 - dispositif d'enseignement / apprentissage

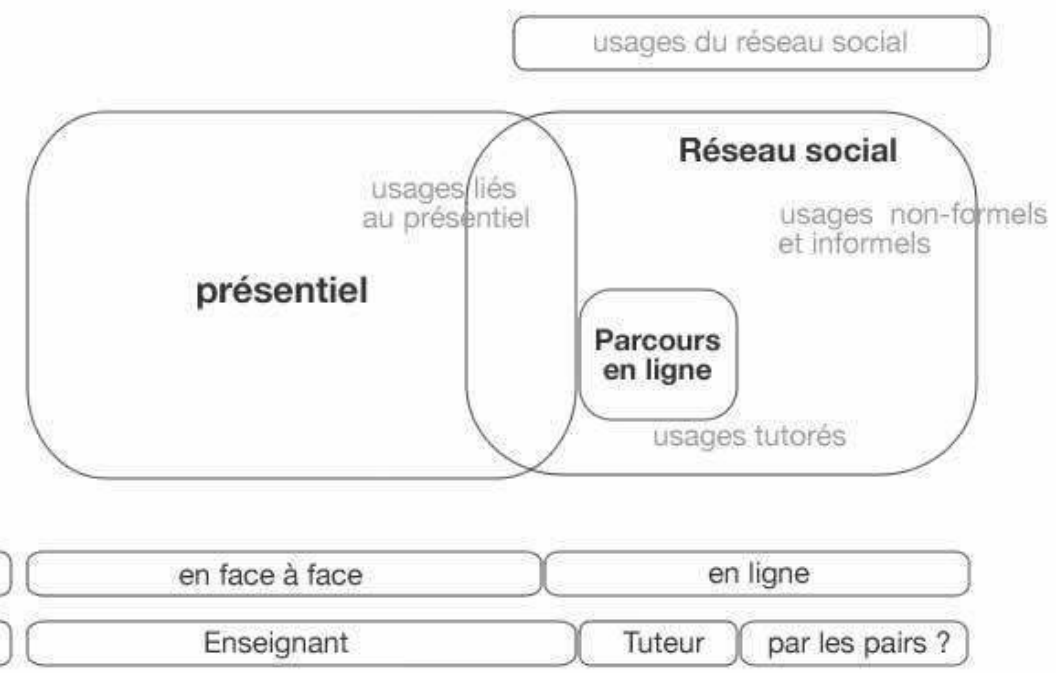

\begin{tabular}{|ccc}
\hline Modalité & en face à face ligne \\
\hline Etayage & Enseignant & Tuteur par les pairs ? \\
\hline
\end{tabular}

Ainsi, le dispositif numérique permet des usages différents du réseau. Il est utilisé pour les cours en ligne tutorés et peut l'être pour des usages liés au cours en présentiel, en 
classe ou à distance. Enfin, il peut être aussi utilisé pour des usages informels. Si le réseau social est ouvert à tous les acteurs du CIEF, dans les faits, peu d'enseignants l'utilisent et donc, il se présente, au niveau des pratiques pédagogiques, comme une enclave dans l'institution.

\section{Le RSN}

Le Réseau Social Numérique est un environnement Ning, accessible à l'adresse http:// rezolumiere.ning.com ${ }_{2}$ ouvert à tous les membres du CIEF. Les inscriptions y sont modérées par un administrateur, l'auteur du présent article. Il constitue un espace collectif de communication et de publication. Le contenu est accessible à tous, en règle générale mais peut être rendu privé. Ning est un RSN qui propose différents outils web 2.0 tels que des outils individuels de publication -photos, vidéos et blogs- et des outils de communication écrite asynchrone (courriels) et synchrone (chat). Il permet aussi la création d'espaces collectifs de travail (outil "groupe"). Chaque membre a une page de profil et un blog, peut créer une liste d'amis et poster des statuts, commenter les activités des autres.

Figure 4 - page d'accueil du réseau social

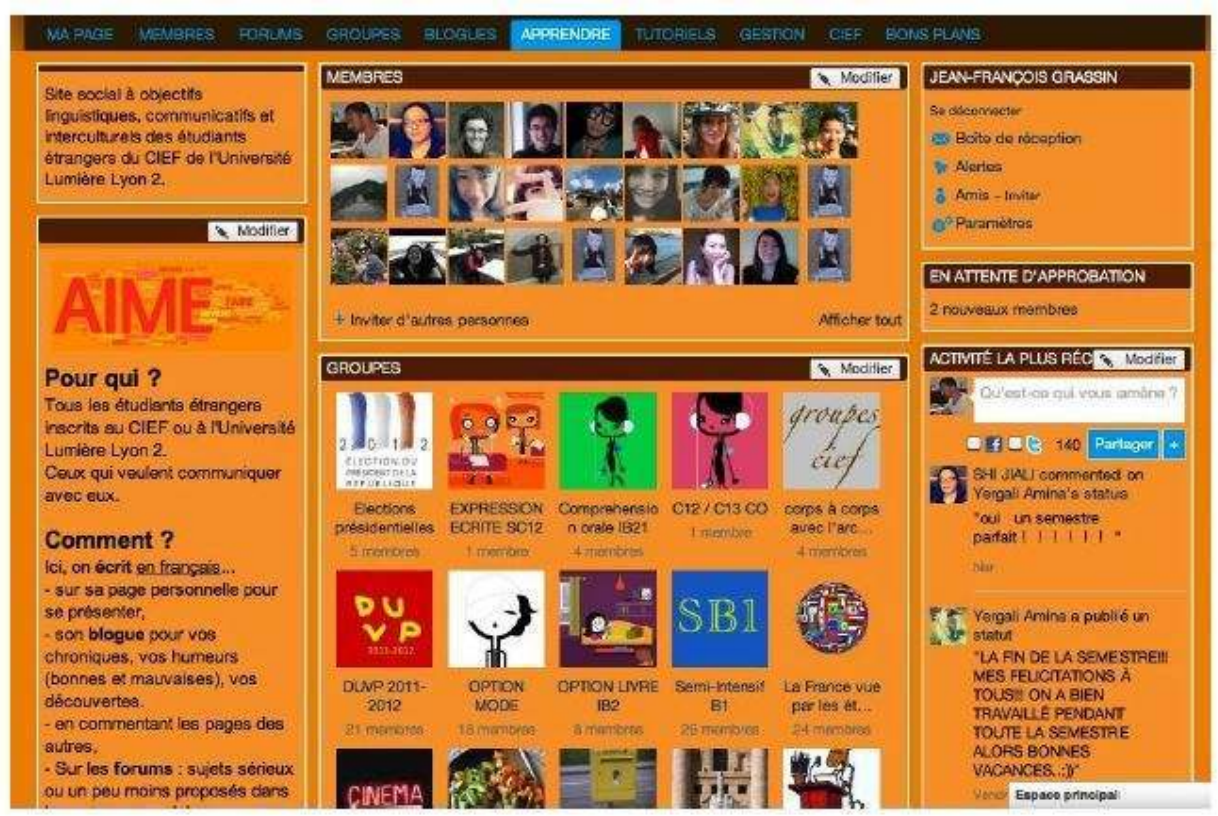

Le RSN, tel qu'il se présente aux utilisateurs (cf. Figure 4), en mettant en exergue le niveau horizontal des membres, et par ses fonctions web 2.0, permet de soutenir l'apprentissage comme un processus social, lui-même soutenu par l'interaction et le dialogue, le partage d'expériences personnelles, le soutien du tuteur et des pairs. Il permet de mettre en valeur les voix personnelles des apprenants en plaçant celle de l'enseignant au même niveau et permet l'accès à des flux d'informations variés. Le dispositif d'apprentissage envisagé ici se veut d'"ethos socio-constructiviste" (Veletsianos \& Navarrete, 2012) en incitant à passer d'une pédagogie du produit à une pédagogie du processus (Albero, 2010). 


\section{Le recueil des données}

Nous avons observé la participation de 59 étudiants répartis en trois groupes égaux, lors d'un semestre de cours, de fin septembre 2011 à mi-janvier 2012. Les données ont été collectées pendant et après le cours. Ce corpus consiste en un recueil des traces écrites sur la plateforme, divers questionnaires en ligne ou sur papier et une série d'entretiens. Les entretiens ont été conduits de manière semi-dirigée. Ces entretiens, d'une durée de 15 minutes à 1 heure ont été menés soit de manière individuelle soit en groupe de 2 à 4 étudiants, principalement pour permettre à des étudiants d'un niveau intermédiaire (B1) de participer à une conversation. Ces entretiens ont été enregistrés et transcrits.

Les questionnaires ont servi à recueillir des informations personnelles sur les usages numériques des participants et sur leur évaluation du RSN et du cours en ligne. Les entretiens ont porté sur leur usage du réseau et leur évaluation de ces activités pour leur apprentissage.

1 De plus, nous avons collecté toutes les traces écrites (productions et interactions) des étudiants sur le réseau. Pour ces données, nous adoptons une analyse de contenu qui nous permet de chercher à identifier les traces de la présence sociale.

Tableau 2 - recueil des données

\begin{tabular}{|l|l|l|l|}
\hline $\begin{array}{l}\text { Procédure de } \\
\text { recueil des } \\
\text { données }\end{array}$ & Contenu & Source & Nombre \\
\hline Début & $\begin{array}{l}\text { Informations basiques sur } \\
\text { l'étudiant et les TIC }\end{array}$ & Questionnaire 1 (en ligne) & 12 \\
\hline Pendant le projet & $\begin{array}{l}\text { Recueil des traces et } \\
\text { productions des étudiants sur la } \\
\text { plateforme }\end{array}$ & $\begin{array}{l}\text { Productions sur tous les } \\
\text { espaces partagés ou individuels } \\
\text { textes et interactions }\end{array}$ & 240 \\
\hline Fin & $\begin{array}{l}\text { Evaluation du dispositif et } \\
\text { réflexions autour des usages }\end{array}$ & $\begin{array}{l}\text { Questionnaires : } \\
\text { - papier } \\
\text {-en ligne } \\
\text { Entretiens individuels et } \\
\text { conjoints }\end{array}$ & 13 \\
\hline
\end{tabular}

\section{L'analyse}

Comme il est de coutume dans une recherche-action, l'auteur était impliqué sur le terrain de l'observation (nous sommes enseignant et tuteur dans l'un de ces groupes). Cela nous a amené à adopter une démarche compréhensive (Dayer \& Charmillot, 2012).

Plusieurs méthodes de triangulation des données ont été mises en place pour maximiser la validité de l'analyse. D'abord, nous collectons des données de plusieurs sources (questionnaires et enquêtes). Ensuite, nous analysons chaque type de données de manière 
indépendante des autres. Pour les entretiens que nous sommes seuls à conduire, nous avons essayé de réduire le biais du rôle duel d'enseignant et chercheur. Nous avons encouragé les participants à une analyse critique et détachée des attentes perçues de notre part.

\section{Premiers résultats et discussion}

44 La première étape de notre analyse repose sur quelques données chiffrées destinées à fournir des repères au chercheur. Nous nous appuyons sur les traces écrites sur le réseau ainsi que sur les questionnaires.

\section{Des usages déclarés}

Les questionnaires soumis aux différents groupes d'étudiants nus ont permis de recueillir des déclarations sur leurs usages du réseau. Le premier graphique montre que la moitié des étudiants ne se connecte que rarement. Seuls, $45 \%$ des étudiants disent se connecter au RSN de manière régulière ou fréquente.

Figure 5 - fréquence de connexion au réseau

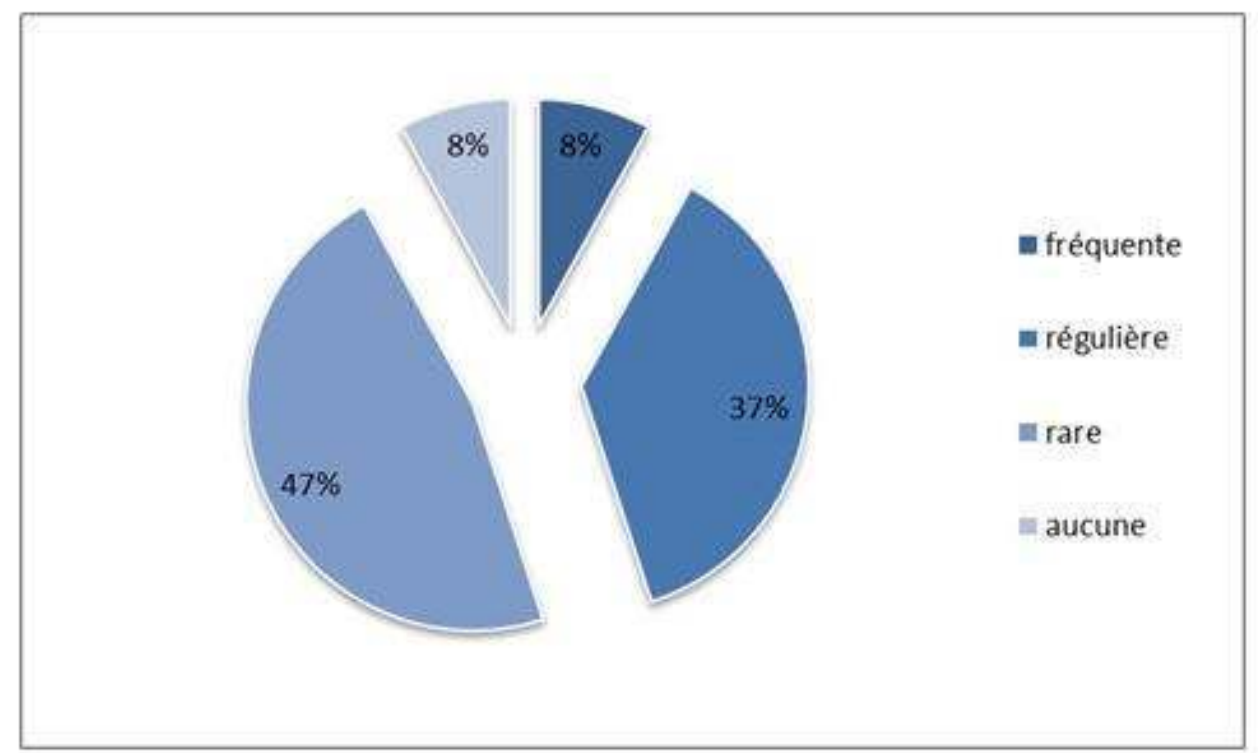

Le deuxième graphique permet de montrer que peu d'activités entraînent un usage régulier, installé, du réseau. La plupart des usages sont occasionnels. 
Figure 6 - activités déclarées par les étudiants

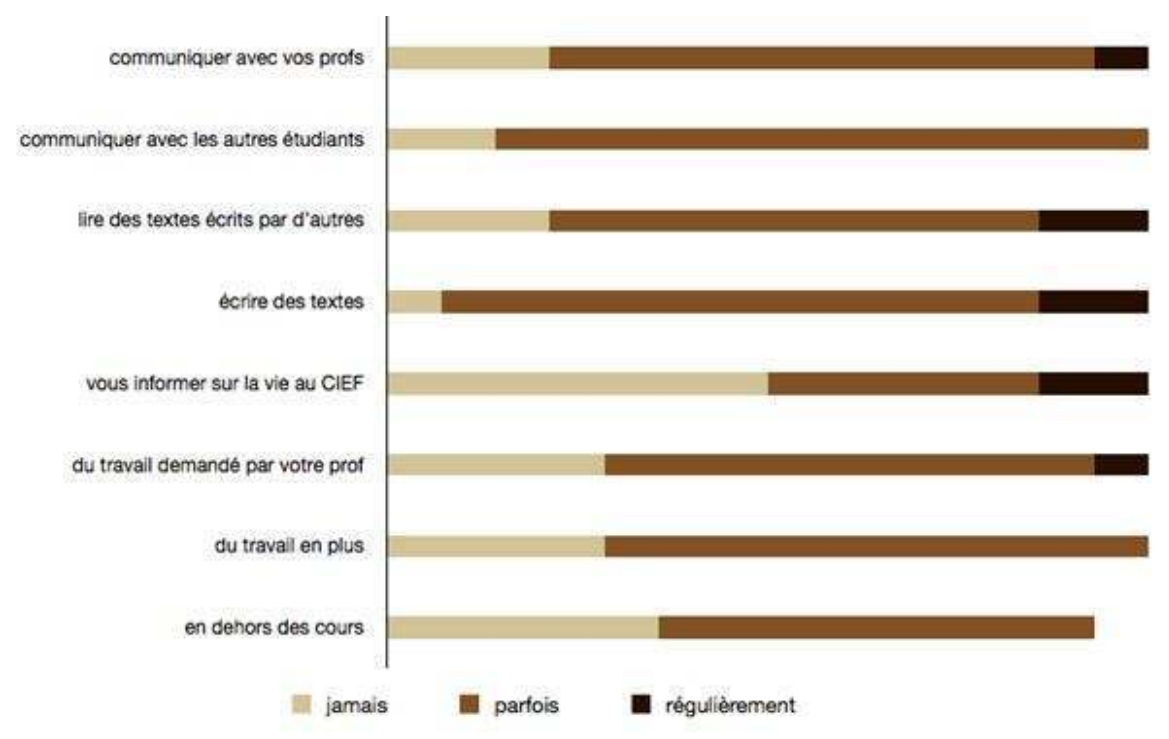

\section{Analyse des traces} 42 ont laissé des traces écrites.

long du semestre. Les pics correspondent à des travaux à rendre pour un cours et les creux à des pauses (ici, les vacances de fin d'année), ce qui indique une utilisation très liée aux cours et peu de maintien du contact.

Nous avons réparti ces traces écrites en trois types. Le premier type ("textes"), qui représente $62 \%$ des productions, correspond à des productions faites dans le cadre d'un cours, à la demande d'un enseignant. Le second type ("parcours") correspond aux productions faites dans le cadre du cours tutoré en ligne et représente $18 \%$ du total. Enfin, le troisième type de traces ("commentaires") correspond aux commentaires informels laissés par les étudiants sur la plateforme. Si ces dernières productions représentent $20 \%$ du total, elles n'émanent que de 18 étudiants sur 59 . Cela veut dire que les deux tiers des étudiants ne sont pas concernés.

\footnotetext{
première est une participation invisible (ce qui ne veut pas dire aucune participation). 8 étudiants n'ont pas laissé de traces sur le réseau mais s'y sont inscrits. Le second type est une participation avant tout éducative, c'est-à-dire que les seules traces laissées sont des traces liées aux cours et à la demande de l'enseignant. Elle correspond à la participation de 28 étudiants. Un autre type serait une participation avant tout sociale, qui repose sur les commentaires et les interactions avec les pairs et les enseignants, mais principalement en dehors de l'injonction de l'enseignant. Celle-ci ne correspond qu'à 2 des étudiants. Enfin, le dernier type serait une participation mixte, où les interactions informelles accompagnent les productions de cours. 13 étudiants adoptent ce type de participation.

A partir des traces recueillies, nous pouvons établir trois types de participation. La
} 
Figure 7 - répartitions des productions écrites suivant le type et dans le temps

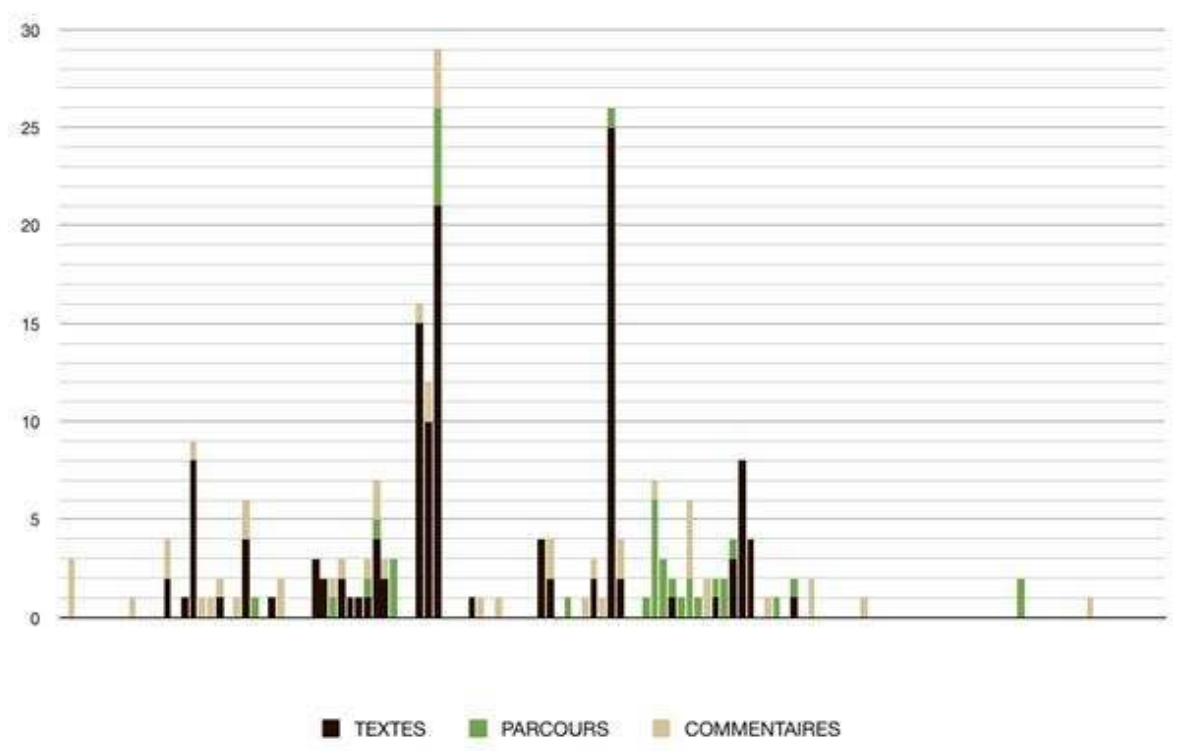

51 Cette différence de participation, qui peut correspondre à des logiques d'engagement différentes dans l'apprentissage mais aussi dans le mode communicatif que propose le réseau, n'est pas surprenante. De la même façon que dans un groupe-classe en face-à-face, tous les membres ne participent pas de la même manière à un cours, l'implication des étudiants en ligne est variable.

Si l'on observe maintenant les profils des étudiants et l'usage de fonctionnalités propres aux réseaux sociaux, nous pouvons voir clairement qu'il y a peu de traces d'appropriation du site en tant que réseau. Si la grande majorité (37) des 51 étudiants inscrits sur le réseau ont mis une photo de profil, les étudiants ne semblent pas adopter le mode de fonctionnement habituel d'un réseau (se construire une liste de contacts pour suivre l'activité de ses "amis" virtuels et communiquer plus facilement avec eux). Les listes d'amis sont réduites : un étudiant a 6 amis en moyenne. 7 étudiants n'ont aucun ami, 21 en ont de 1 à 9 et enfin, 9 étudiants ont une liste d'amis supérieure à 10. Ce sont des chiffres modestes si l'on pense à la taille du groupe-classe en présentiel (18 étudiants). Enfin, l'usage de la fonction "j'aime" est quasiment absente ; la fonction qui permet de créer un lien de sa production (ou de son statut) à partir du réseau institutionnel vers un réseau personnel (type Facebook) n'a été employé que par 2 étudiants.

Malgré le peu de participation, les marques de la présence sociale sont observables. Si nous analysons les productions, nous percevons les 2 premières étapes identifiées par Cuissi : le dévoilement de soi et la construction de liens socio-affectifs. La place nous manque ici pour détailler l'analyse de ces marques, mais cela confirme l'idée qu'

une nouvelle génération d'utilisateurs est capable de vivre sa socialisation dans un espace virtuel dans lequel le lien technologique peut être porteur d'engagement émotionnels et de phénomènes sociaux au même titre que la vie sociale traditionnelle (Ciussi, 2007).

Mais cette actualisation du potentiel pour l'apprentissage en langue reste marginale et reste principalement dépendante des usages prescrits par l'enseignant.

L'essentiel des interactions est sur le modèle "object-centered sociality" (cf. supra 1.3), la conversation autour d'objets, ici des photos. Dans ces occasions de participation, nous retrouvons les signes de la présence sociale et cela nous paraît être une situation de 
communication à penser pour l'apprentissage car elle parait évidente à la plupart des étudiants. Les conversations autour de photos et de vidéos en français peuvent donner lieu à des échanges interpersonnels dans lesquels même des étudiants qui disposent de faibles ressources langagières peuvent s'engager.

De même, la plupart des activités de négociation du sens se font à partir de ce modèle de communication et de socialité. La copie d'écran ci-dessous (Figure 8) montre ainsi deux étudiantes commenter la photo de l'une d'entre elles et négocier le sens de l'activité.

Figure 8 - négociation de sens autour d'un objet d'apprentissage

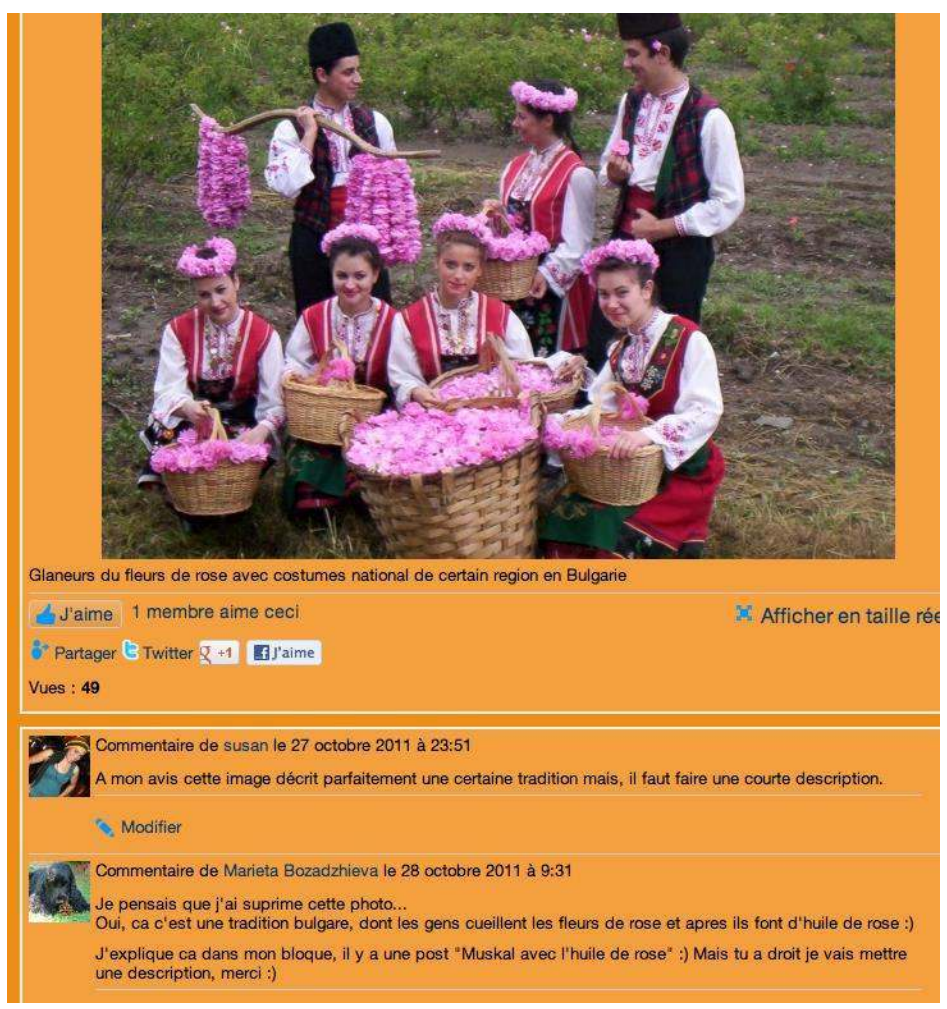

Les activités des étudiants se limitent donc généralement aux tâches assignées. Fluckiger (2011) constataient déjà que, "si les étudiants communiquent beaucoup en général, ils le font assez peu en contexte éducatif". Cela témoigne des difficultés d'intégration des formes participatives d'enseignement en ligne dans le supérieur. Alors que la communication sur des réseaux sociaux comme Facebook correspond pour les étudiants à une communication quotidienne et routinière avec un grand nombre de correspondants, la communication avec les autres étudiants sur les réseaux institutionnels est peu fréquente et avec un nombre très limité de partenaires.

Les étudiants ont utilisé l'environnement d'apprentissage d'une manière essentiellement éducative, dans ces espaces les plus formels et intentionnels, laissant la plupart du temps de côté les espaces où l'apprentissage se révèlerait moins formel et les occasions plus inattendues. Ainsi, le réseau est rapidement déserté en fin de semestre par les étudiants qui s'y connectaient auparavant, si aucun enseignant n'interagit avec eux dans cet environnement. Dans les entretiens, les étudiants déclarent en effet n'y être allés que très rarement après le mois de janvier.

59 Pour la plupart des traces écrites, il s'agit d'échanges informationnels prescrits par l'enseignant. La publication sur le réseau se fait sur injonction de l'enseignant et donc, 
dans l'esprit de la majorité des étudiants, n'implique pas de réponse. Les outils du web 2.0 sont à disposition mais nous constatons une permanence de la communication verticale ; la plupart des étudiants ne profitent pas de la communication horizontale. Seules, quelques échanges de commentaires ou de statuts dépassent le $2^{\mathrm{e}}$ tour de parole ${ }^{2}$. Des tentatives d'interaction sociale sont présentes mais s'interrompent vite. L'absence de réponse est perçue comme une absence de volonté de communiquer et l'espace informel de ces interactions est abandonné ou devient le lieu de connexions très ponctuelles. Ainsi, une étudiante lors d'un entretien nous dit:

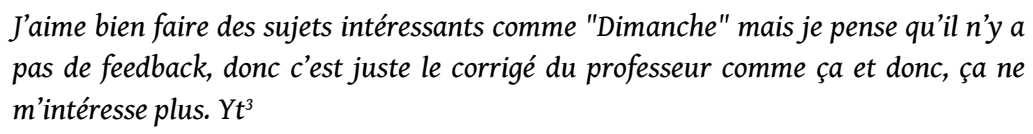

Cette remarque montre aussi la différence de statut entre l'écrit dans la classe et l'écrit produit sur le réseau. L'interaction est, au départ, perçue comme inhérente à la communication en réseau. Or, cette dimension est rarement prise en compte par l'enseignant. La production écrite sur le réseau est souvent gérée de la même manière que la production écrite en classe. Sans doute peut-on dire que les enseignants n'ont pas assez planifié d'activités collectives en ligne pour que les interactions soient perçues comme essentielles à l'apprentissage et que la construction d'une communauté soit un enjeu.

\section{Des perceptions mitigées}

\section{Un RSN perçu comme utile à l'apprentissage}

61 En règle générale, le réseau est perçu comme utile. Les obstacles énoncés sont avant tout le temps et le manque d'intérêt pour les sujets proposés ainsi que des difficultés de connexion personnelle (cf. Figure 9).

86\% des étudiants le trouvent utile pour leur apprentissage du français. 73\% des étudiants se disent motivés à communiquer sur le réseau avec les autres membres, $72 \%$ motivés à écrire en voyant les textes des autres, $77 \%$ par pouvoir lire le texte des autres et comparer leurs propres écrits en début et en fin d'apprentissage, avec ceux des autres. 
Figure 9 - obstacles déclarés au travail sur le réseau

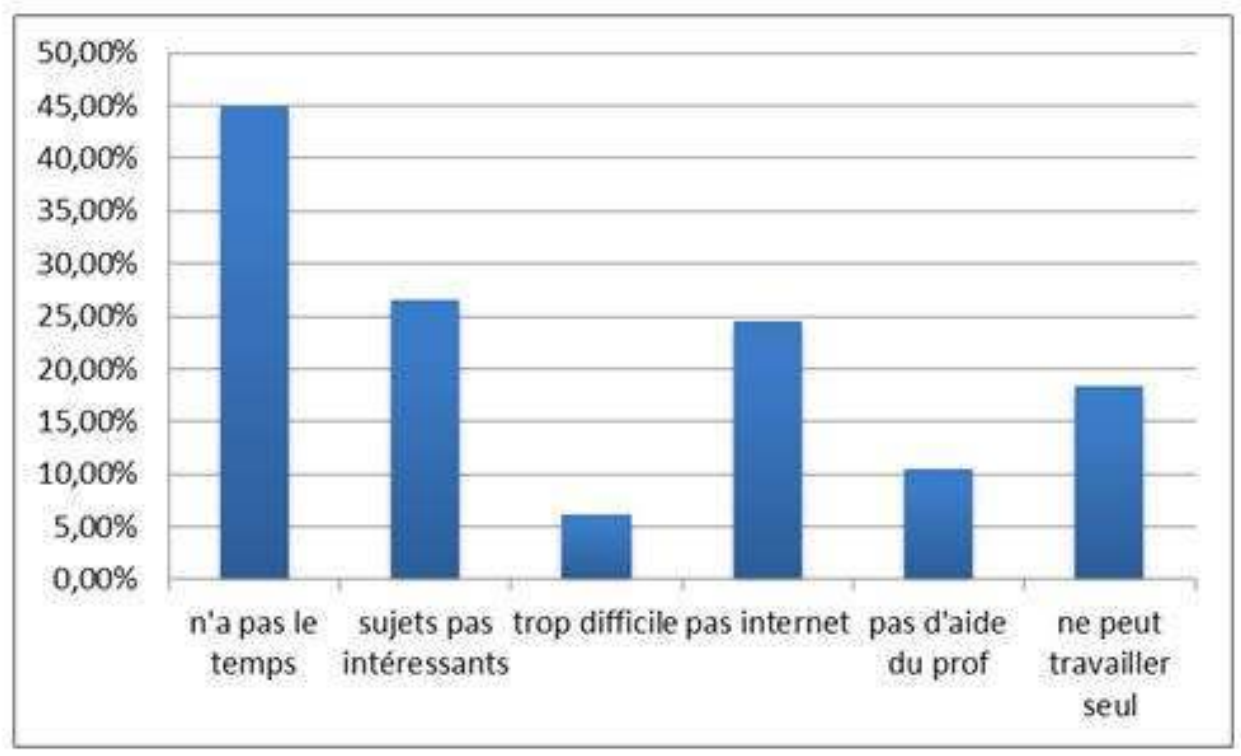

\title{
UN RSN perçu comme inefficace à connecter les gens entre eux
}

À l'usage, le site est plus utilisé comme un site d'accès à des ressources pour le travail d'un cours que comme une plateforme relationnelle. Pourtant, la plupart des étudiants interrogés perçoivent ce site au départ comme un RSN au même titre que leurs réseaux personnels. Il est vu d'abord comme un site pour se construire un réseau d'amis, comme en témoignent ces étudiants lors des entretiens que nous avons menés :

\begin{abstract}
Parce qu'en fait, c'est le début de mon installation à Lyon 2 et je vais voir mon groupe car je ne connais pas beaucoup de gens. J'aurais pu parler avec mes nouveaux camarades, savoir plus parce que je n'ai pas leur contact et c'est tout et j'ai regardé le chat, il y avait juste une fille avec qui je parle et qui était dans mon groupe, As.

On peut lui [à l'étudiant] dire, c'est un outil, peut-être on peut se faire des amis, parce que nous sommes nouveaux, on ne connait pas beaucoup de gens, on peut sur Rezo Lumière ${ }^{4}(R L)$ pour faire connaissance. We
\end{abstract}

Mais le petit nombre d'étudiants présents sur le réseau, le peu de flux dynamiques et quotidiens d'informations nouvelles sur les autres, rendent les connexions entre les membres trop rares et, déçus, les étudiants se tournent rapidement vers d'autres artefacts, jugés plus efficaces.

\footnotetext{
Je n'ai pas utilisé le réseau pour communiquer. Il fallait que ce soit habituel et on n'avait pas l'habitude. on connait bien le site social FACEBOOK qui est plus habituel pour l'instant pour nous. [...] En général, on peut parler de ce qui nous attire pour être sur un site social, c'est que on voit des amis qui nous intéresse, qu'on peut communiquer avec eux librement et ici, dans RL, on a peu de gens qu'on connait et pour créer une conversation tout ça, ça va être plus compliqué. Gu

J'ai utilisé le RL le dernier semestre mais c'est juste pour faire les devoirs de votre cours. Mais pour communiquer, j'ai utilisé le Facebook mais en français avec mes amis ; parce que sur le RL, il n'y a pas beaucoup de personnes ; dans le chat oui, peut-être juste une ou deux, c'est vraiment pas beaucoup. Yt
} 
La déception est là pourtant les étudiants font la différence entre Facebook, le réseau social que la plupart utilise pour des usages sociaux et un réseau social pour l'apprentissage. Ils semblent reconnaître l'utilité des deux, dans des sphères différentes mais qui peuvent aussi être poreuses :

\begin{abstract}
Je voulais travailler beaucoup sur ce site je ne sais pas c'est mon opinion mais ça m'intéresse de parler avec mes camarades et je voulais travailler. Par exemple, si je mets une vidéo de mon pays etc., je parle ou je cherche un logement, je fais beaucoup d'exercices et il y a des camarades avec qui je parle, ça m'intéresse à parler. Ils peuvent commenter mes vidéos ou dire quelque chose de son pays et en comparaison de la France et c'était plus intéressant pour moi. Parce qu'il y a beaucoup de gens que je ne connais pas et c'est pas intéressant pour moi. Par exemple, sur Facebook, je parle avec des gens que je connais. Il y a beaucoup de gens de camarades de mon groupe qui sont sur Facebook. Mais c'est intéressant pour moi s'ils ont été sur le réseau car sur Facebook, c'est juste une chose pas important pour mes études et pour comprendre le français mais ici oui. Et quand j'étais ici, j'ai dit au début, il n'y avait pas mon groupe et je ne savais pas ce que je devais faire. Il y avait beaucoup de mes camarades mais il n'y avait pas de groupe s1b4. As
\end{abstract}

Le site demande en outre un effort d'appropriation pour certains étudiants :

La première fois, j'ai trouvé que c'était difficile parce que je n'aime pas beaucoup la technologie peut-être mais après, quand je l'ai utilisé plus, j'ai trouvé que c'était bien organisé. Su

Il faut donc que l'effort en vaille la peine, le réseau doit s'agrandir :

Je propose que tous les étudiants peuvent s'inscrire dans ce site et tous les professeurs peuvent proposer des devoirs sur ce site. Si on l'utilise très courant, je pense il va être mieux. Mais je pense qu'ils ont besoin de temps. Dx

Lorsqu'on les interroge sur l'amélioration de la plate-forme, les étudiants pensent tout de suite au relationnel. Ensuite, l'intérêt du réseau est l'apprentissage de la langue ; mais entrer en interaction avec les autres est souvent la première motivation.

On peut lui (l'étudiant) dire, c'est un outil, peut-être on peut se faire des amis, parce que nous sommes nouveaux, on ne connait pas beaucoup de gens, on peut aller sur RL pour faire connaissance. [...] A Lyon, je pense que c'est la chose attractive [...] mais après on peut demander du travail sur RL, quand il y a plus d'étudiants qui utilisent ça. [...] D'abord, il faut attirer les nouveaux étudiants. We

68 Les étudiants s'intéressent d'abord aux membres du réseau qu'ils connaissent ${ }^{5}$. Cette vision de l'utilité du réseau -mettre en contact les membres les uns avec les autreschangent cependant avec le temps. Ainsi cet étudiant explique ne pas voir le même intérêt pour le réseau d'un semestre à l'autre :

Ca dépend du semestre car le 1er on peut utiliser le site du RL mais au 2e on l'utilise moins qu'avant car au 1er semestre, c'est important d'apprendre le français par ce que la plupart des étudiants vient en septembre ou en août mais au 2nd semestre, la situation a un peu changé. Donc on a beaucoup d'amis entre les groupes, et après avoir fait des amis, on utilise le Facebook ou le Twitter pour se communiquer. Th

69 Enfin, nous remarquons aussi des motivations différentes suivant les étudiants. Ainsi, cette étudiante est motivée d'abord par la communication, puis par le travail, 
Je crois que ça doit être présenté comme un site réseau pour avoir relation avec

d'autres personnes et après introduire qu'il est aussi pour le travail. Su

tandis que d'autres entreront sur le réseau pour travailler et en profiteront pour entrer en contact.

J'ai utilisé en fait c'est juste pour améliorer ma grammaire. Il y a un très bon exercice. Puis j'ai cherché des Oügour ${ }^{6}$ mais malheureusement, c'est seulement moi. Dil l'enseignant, du moins au début de leur utilisation du site :

Moi, je pense qu'il faut commencer par-là, pour que tous les profs soient dans le réseau. Gu

Si on entre directement la page Rezo, on ne sait pas qu'est-ce qu'on peut faire, je crois qu'on a besoin d'une petite explication. $\mathrm{Su}$

\section{Conclusion et pistes de recherche}

71 L'analyse du dispositif atteste certes des potentialités pour l'apprentissage des langues mais celles-ci ne sont que rarement actualisées. L'engagement est peu important : même si les étudiants identifient immédiatement l'environnement comme un réseau social, les usages ne sont pas réguliers mais avant tout répondent à la prescription de l'enseignant. De même, la pédagogisation du site semble ne pas correspondre à cette architecture de la participation, au fonctionnement en réseau et à des activités conduites autour du profil des membres.

On peut tout d'abord repérer comme cause première à ce relatif échec, le décalage entre usages sociaux et usages pédagogiques. En effet, il faut prendre conscience que "l'institution éducative intègre chaque nouvel objet en fonction de ses modes habituels d'activité et ses finalités propres indépendamment des particularités de celui-ci et de ses usages dans le contexte social" (Albero, 2010).

Cela aboutit à un détournement de l'artefact en réduisant ses potentiels les plus perturbants pour le système. Ni les enseignants ni l'institution ne semblent avoir intégré la dimension "réseau" dans leurs pratiques car les étudiants perçoivent la demande qui émane de ces acteurs du système comme relevant d'une communication verticale, avant tout informationnelle, par la proposition de situations d'apprentissage soit tournées vers l'input (le site permet d'accéder à des ressources), soit tournées vers l'output (le site permet de publier des productions), mais peu interactionnelle et dynamique. Le potentiel du réseau pour l'apprentissage est peu perçu par les acteurs ; elle ne peut donc être mise en œuvre par les étudiants qui restent "perdu dans l'espace social", selon la formule de Dron et Anderson (2009). Or, il faut un guidage pour qu'elle se concrétise au niveau de la communauté. L'implémentation d'un dispositif socio-techno-pédagogique demande que les acteurs développent une compréhension appropriée de la pédagogie reliée pour s'y engager activement.

Du côté des étudiants, une approche serait de fournir des attentes pédagogiques explicites quant à la construction de la communauté et de son potentiel pour l'apprentissage (Halic et al., 2012). Valoriser le potentiel éducatif de ces dispositifs en 
précisant les modalités d'accompagnement pédagogiques pour éviter qu'ils restent en marge de la formation ou réservés aux étudiants les plus compétents, en terme techniques mais aussi et surtout en termes d'enjeux d'apprentissage (Rivens Mompean, 2011). Cela demande de mettre en exergue une vision de l'apprentissage au sein d'une "épaisseur sociale" car l'appropriation de la langue comme du dispositif implique que le maintien du lien social soit un enjeu fort de l'apprentissage. L'appropriation ne se fait pas en dehors du social. Pour que le web social soit déclencheur de pratiques d'apprentissage, il faut accompagner l'étudiant dans son processus d'appropriation et donc, dans des stratégies qui le conduisent à une certaine distance cognitive et l'incitent à la réflexivité (Rivens Mompean, 2011).

$\mathrm{Du}$ côté de l'enseignant, ce type de dispositif techno-pédagogique nécessite des compétences organisationnelles et communicationnelles spécifiques. On assiste à une complexification de l'acte formatif par de nouveaux modes relationnels, d'écriture et temporels (Rivens Mompean, 2011) qui rendent difficile son intégration à l'arsenal pédagogique. Intégrer le web social à un dispositif d'enseignement/apprentissage, c'est intégrer une architecture de la participation qui implique la production continuelle de contenus et l'aspect viral ${ }^{7} \mathrm{du}$ web. Pour l'enseignant, cela demande de planifier des épisodes d'apprentissage qui utilisent le réseau à la fois comme une plateforme de socialisation et d'apprentissage. Ces enjeux de ce web social ne sont pas encore assez pris en compte dans la conception de nos cours. "Dans ce contexte, nos environnements d'apprentissage, avec leur vision prescriptive et descendante de l'apprentissage, apparaissent contraignants, limités et décalés" (Henri, 2011 : 624). Cependant, il faut prendre conscience que, pour l'enseignant, le web social correspond à une prise de risque puisque les interactions y sont moins prévisibles et planifiables.

Enfin, le dispositif techno-pédagogique de type réseau social souffre de n'être qu'une enclave institutionnelle. Il est indispensable qu'il soit ancré aux trois niveaux : le niveau macro, institutionnel -quel est le discours de l'institution à l'égard de ce dispositif ?-, le niveau méso, celui du dispositif pratique -comment apparait-il dans les scénarios d'apprentissage conçus par les enseignants ?- et au niveau micro, celui de l'activité de l'apprenant.

77 Deux pistes de recherche s'offrent à nous pour continuer cette réflexion. D'abord, il nous faut nous pencher maintenant sur les perceptions, les représentations et les usages des autres acteurs, l'institution et les enseignants. Ensuite, il sera intéressant de tester le potentiel de certaines tâches sur le réseau et leur potentiel pour l'apprentissage de façon à mieux cerner les apports d'un tel dispositif. Le but de notre travail est de continuer à questionner le potentiel d'un RSN pour l'apprentissage des langues.

\section{BIBLIOGRAPHIE}

Albero, B. (2010). "Penser le rapport entre formation et objets techniques. Repères conceptuels et épistémologiques". In Leclerc, G. et Varga, R. Dispositifs de formation et environnements numériques : enjeux pédagogiques et contraintes informatiques. Paris : Hermès / Lavoisier, pp. 38-69. 
Anis, J. (1998). Texte et ordinateur. L'écriture réinventée ? Paris-Bruxelles : De Boeck Université.

Arnold, N. \& Paulus, T. M. (2010). "Using a social networking site for experiential learning: Lurking, modeling and community building" The Internet and Higher Education, vol. 13, $\mathrm{n}^{\circ} 4$. pp. 188-196.

Audran, J. \& Simonian, S. (2009). "Etudier les communautés d'apprenants en ligne : quel(s) agencement(s) des méthodes de recherche ? ". Education \& Formation, nº-290. http:// ute3.umh.ac.be/revues/index.php?revue $=6 \&$ page $=3$

Boyd, S. (2005). "Are you ready for social software?" Darwin Magazine. http://stoweboyd.com/ post/2325281845/are-you-ready-for-social-software

Boyd, D. \& Ellison, N. (2007). "Social network Sites : definition, History and scholarship". Journal of Computer Mediated Communication, vol. $13, \mathrm{n}^{\circ} 1$.

Cardon, D., Crepel, M., Hatt, B., Pissard N. \& Prieur C. (2008). "10 propriétés de la force des coopérations faible", Internet actu.net. http://www.internetactu.net/2008/02/08/10-proprietesde-la-force-des-cooperations-faible/

Ciussi, M. (2007). "Dynamique des liens sociaux à distance : genèse des formes et processus observables", Symposium "Processus de socialisation et apprentissages en ligne", Congrès internationalAREF. http://www.congresintaref.org/actes_pdf/ AREF2007_Melanie_CIUSSI_BOS_125.pdf

Conner, M. (1997-2013). "Informal learning".

http://marciaconner.com/resources/informal-learning/

Dabbagh, N. \& Kitsantas, A. (2012). "Personal Learning Environments, social media, and selfregulated learning : a natural formula for connecting formal and informal Learning". The Internet and Higher Education, vol. 15, $\mathrm{n}^{\circ} 2$, pp. 3-8.

Dayer, C. \& Charmillot, M. (2012). "La démarche compréhensive comme moyen de construire une identité de la recherche dans les instituts de formation". Formation et pratiques d'enseignement en questions $\mathrm{n}^{\circ} 14$, pp. 163-174. http://www.revuedeshep.ch/site-fpeq/

Site_FPEQ/14_files/11_dayer.pdf

Dron, J., \& Anderson, T. (2009). "Lost in social space: Information retrieval issues in Web 1.5". Journal of Digital Information, vol. 10, $\mathrm{n}^{\circ} 2$. pp. 1-12.

Fluckiger, C. (2011). "De l'émergence de nouvelles formes de distance. Les conséquences des nouvelles pratiques de communication ordinaires sur la FAD dans le supérieur", Distances et Savoirs, vol. 9, $\mathrm{n}^{\circ}$ 3. pp. 397-417.

Garrison, R., Anderson, T., \& Archer, W. (2000). "Critical inquiry in a text-based environment : Computer conferencing in higher éducation". The Internet and Higher Education, vol. 2, $\mathrm{n}^{\circ} 2-3$. pp. 87-105.

Granovetter, M. S. (1973). "The strengh of weak ties". American Journal of Sociology. vol. 78, $\mathrm{n}^{\circ} 6$. pp. 1360-1380.

Guichon, N. (2011) Apprentissage des langues médiatisé par les technologies : contribution à l'épistémologie de la didactique des langues, vol. 1. Dossier présenté pour l'HDR. Le Havre : Université du Havre.

Halic, O. et al (2010). "To blog or not to blog : Student perceptions of blog effectiveness for learning in a college-level course", The Internet and Higher Education, vol. 13, n 4. pp. 206-213. 
Henri, F. (2011) "Où va la distance ? Est-ce la bonne question ?". Distances et savoirs, vol. 9, n 4. pp. 619-663.

Henri, F. \& Pudelko, B. (2006). "Le concept de communauté virtuelle dans une perspective d'apprentissage social". In A. Daele et B. Charlier (éds). Comprendre les communautés virtuelles d'enseignants : pratiques et recherches. Paris : L' Harmattan. pp. 105-123.

Ito, M. (dir.) 2008. Living and learning with new media: summary of findings from the digital youth project. Berkeley. http://digitalyouth.ischool.berkeley.edu/fi les/report/digitalyouthWhitePaper.pdf

Kabilan et al. (2010). "Facebook : An online environment for learning of English in institutions of higher éducation". The Internet and Higher Education, vol. 13, n 4. pp. 179-187.

Lee, M. J. W. \& McLoughlin, C. (2010). "Beyond distance and time constraints: Applying social networking tools and Web 2.0 approaches in distance education". In G. Veletsianos (dir.), Emerging Technologies in Distance Education, Athabasca : Athabasca University Press. pp. 61-87. http://www.aupress.ca/books/120177/ebook/04_Veletsianos_2010-

Emerging_Technologies_in_Distance_Education.pdf

Mangenot, F. (2011). "Apprentissages formels et informels, autonomie et guidage", Symposium "Web social et communautés autour des langues étrangères : la part du formel et de l'informel". In Dejean, Mangenot, Soubrié (dir.) Actes du colloque Echanger pour apprendre en ligne (EPAL). http://w3.u-grenoble3.fr/epal/actes.html

Mondada, L. \& Pekarek Doehler, S. (2000). "Interaction sociale et cognition située : quels modèles pour la recherche sur l'acquisition des langues? ". Acquisition et interaction en langue étrangère, vol. 12. http://aile.revues.org/947

Pakdel, A. \& Springer, C. (2010). "De l'activité communicative à l'activité sociale en ligne : exploiter les réseaux sociaux pour élargir le cadre social de la classe de langue." Actes du colloque "Languages, cultures and virtuals communities", Eurocall Bordeaux.

Pekarek Doehler, S. (2000). "Approches interactionnistes de l'acquisition des langues étrangères : concepts, recherches, perspectives". Acquisition et interaction en langue étrangère, vol. 12. http:// aile.revues.org/934

Peraya, D. (2011). "Un regard sur la "distance", vue de la "présence" ", Distances et savoirs, vol. 9, n 3. pp. 445-452.

Rivens Mompean, A. (2011). "Distances plurielles pour l'apprentissage des langues", Distances et savoirs, vol. $9, \mathrm{n}^{\circ}$ 3. pp. 375-396.

Shirky, C. (2003). A group is its own worst enemy: Social structure in social software. O'Reilly Emerging Technology conference, Santa Clara, CA, 24 avril 2003. http://www.shirky.com/ writings/group_enemy.html

Stenger, T. \& Coutant, A. (2010). "Les Réseaux sociaux numériques : des discours de promotion à la définition d'un objet et d'une méthodologie de recherche". Hermès - Journal of Language and Communication Studies, $\mathrm{n}^{\circ}$ 44. pp. 209-228.

Tricot, A. (2011). Apprentissages et documents numériques. Paris : Belin.

Veletsianos, G. \& Navarrete, C. (2012). "Online Social Networks as Formal Learning Environments: Learner Experiences and Activities". The International Review Of Research In Open And Distance Learning, vol. 13, n 1. pp. 144-166 http://www.irrodl.org/index.php/irrodl/article/ view/1078/2077 
Wenger, E. (1998). Communities of Practice. Cambridge : CUP.

Wodzicki, K. et al. (2012). "Actually, I wanted to learn" : study-related knowledge exchange on social media". The Internet and Higher Education, vol. 15, $\mathrm{n}^{\circ} 1$. pp. 9-14

Zourou, K. (2012). "De l'attrait des médias sociaux pour l'apprentissage des langues - Regard sur l'état de l'art", Alsic , vol. 15, n 1. http://alsic.revues.org/2485

\section{NOTES}

1. Selon Mangenot (2011).

2. Ce qui est constaté dans les forums de discussion en ligne par de nombreux auteurs (cf. Audran \& Simonian, 2009).

3. Les citations sont tirées des entretiens avec les étudiants, retranscrits ici sous forme de verbatim.

4. Rezo Lumière, nom du réseau social du CIEF.

5. La qualité de l'interface du site peut être aussi remise en cause : il n'y pas assez d'interactivité et de dynamisme. La page d'accueil est trop statique, elle ne montre pas de flux personnalisés ceux des amis dont on suit l'activité et limite de fait l'augmentation de la prise de conscience périphérique ("enhancement of peripherical awareness") repérée par Boyd (Zourou, 2012).

6. L'étudiante cherchait des personnes de même origine qu'elle.

7. Nous entendons par "aspect viral" avant tout la circulation horizontale de l'information et de la connaissance et la visibilité comme opportunité de coopération (voir pour plus de détails Cardon 2008).

\section{RÉSUMÉS}

Cette étude porte sur l'intégration d'un réseau social numérique au sein d'un département universitaire d'enseignement du Français Langue Etrangère en France et cherche à identifier les conditions de l'actualisation de ses potentialités pour l'apprentissage. Nous examinons les usages que font 59 étudiants de ce réseau social et leurs représentations de ce site reliées à leur apprentissage de la langue. Notre analyse montre qu'il y a peu de traces d'appropriation du site en tant que réseau, mais que malgré le peu de participation, les marques de la présence sociale sont observables. Même si les étudiants identifient immédiatement l'environnement comme un réseau social, les usages ne sont pas réguliers mais avant tout répondent à la prescription de l'enseignant. Le site est plus utilisé comme un site de ressources pour le cours que comme une plateforme relationnelle. Son utilisation pédagogique semble ne pas correspondre aux objectifs de l'apprentissage en réseau. Les enjeux du web social ne sont pas assez pris en compte dans la conception des cours.

This study focuses on the integration of a digital social network within a university department that teaches French as a foreign language in France and seeks to identify the conditions for the actualization of its potentialities for learning. We examine usage of the social network by 59 students and their perception of this site related to their language learning. Our analysis shows that there is little evidence of appropriation of the site as a network, but despite the lack of 
participation, traces of social presence are present. Although students immediately recognize the environment as a social network, usage is not regular but above all responds to the requirement of the teacher. The site is used more as a resource site for the course than as a relational platform. Its educational use does not seem to correspond to the learning objectives in a network. Issues of the social web are not sufficiently taken into account in the course design.

INDEX

Mots-clés : réseaux sociaux, médias sociaux, apprentissage en ligne, usages, représentations Keywords : social learning networks, social media, online learning, uses, representations

\section{AUTEUR}

\section{JEAN-FRANÇOIS GRASSIN}

Jean-François Grassin est professeur certifié au Centre International d'Etudes Françaises de l'Université Lumière Lyon 2, doctorant au laboratoire ICAR sous la direction de Nicolas Guichon, professeur à l'Université Lumière Lyon 2. Il s'intéresse à l'intégration des TIC dans

l'enseignement et pour l'apprentissage des langues et en particulier aux potentiels des réseaux sociaux et des outils en ligne du web 2.0.

Courriel : jean-francois.grassin@univ-lyon2.fr

Adresse : Centre International d'Etudes Françaises, Université Lumière Lyon 2, 16 Quai Claude Bernard, 69365 Lyon cedex 07 